\title{
Crystal structure of tricaesium arsenide, $\mathrm{Cs}_{3} \mathrm{As}$
}

\author{
H. Hirt and H. J. Deiseroth* \\ Universität Siegen, Anorganische Chemie, D-57068 Siegen, Germany
}

Received October 28, 2002, accepted and available on-line January 11, 2002; CSD-No. 409668

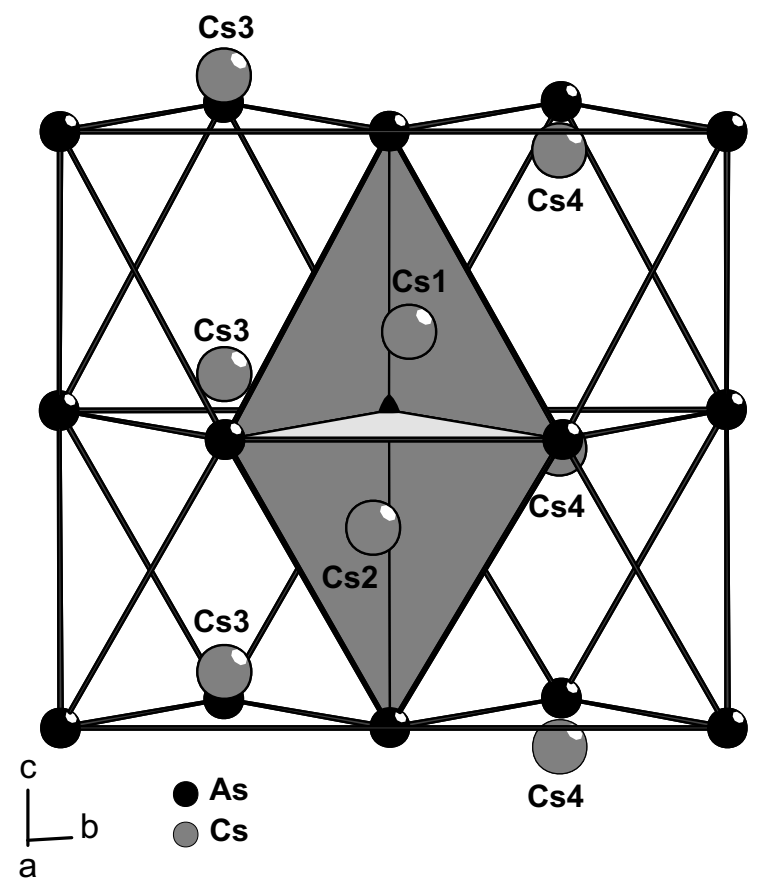

Abstract

$\mathrm{AsCs}_{3}$, hexagonal, $P 6_{3} \mathrm{~cm}$ (No. 185), $a=10.783(2) \AA$, $c=11.147(2) \AA, V=1122.5 \AA^{3}, Z=6, R_{\mathrm{gt}}(F)=0.031$, $w R_{\mathrm{ref}}\left(F^{2}\right)=0.059, T=293 \mathrm{~K}$.

\section{Source of material}

The compound was synthesized from a stoichiometric mixture of the elements in a sealed steel-tube filled with inert gas (Ar). The sample was first heated up to a homogeneous melt at $670 \mathrm{~K}$ (1d). Well shaped, dark grey single crystals of the air sensitive compound were obtained after subsequent annealing at $540 \mathrm{~K}$ (four weeks).

\section{Experimental details}

Refinements assuming the space groups $P \overline{6} c 2$ and $P 6_{3} / \mathrm{mcm}$ yielded higher $R_{\mathrm{gt}}(F)$ values (0.107 and 0.188 , respectively).

\section{Discussion}

The compound was first mentioned by Gnutzmann [1] without assignement of a space group but excluding $P 6_{3} / m m c$ [2]. $\mathrm{Cs}_{3} \mathrm{As}$ crystallize in the $\mathrm{Na}_{3} \mathrm{As}$ structure [3] $\left(\mathrm{Cu}_{3} \mathrm{P}\right.$ type $)$ based on a hexagonal close packing of arsenic $\left(\mathrm{As}^{3-}\right)$. All tetrahedral holes are filled with $\mathrm{Cs}^{+}$off-centered towards a tetrahedral faces (Cs2) and towards a tetrahedral edge (Cs1). The cesium-atoms Cs3 and Cs4 (see figure) are strongly shifted along [001] away from the centres of the trigonal holes.

Table 1. Data collection and handling.

$\begin{array}{ll}\text { Crystal: } & \begin{array}{l}\text { dark grey metallic needle, } \\ \text { size } 0.05 \times 0.05 \times 0.2 \mathrm{~mm}\end{array} \\ & \text { Mo } K_{\alpha} \text { radiation }(0.71073 \AA) \\ \text { Wavelength: } & 187.95 \mathrm{~cm}^{-1} \\ \mu: & \text { Stoe IPDS, } \varphi \\ \text { Diffractometer, scan mode: } & 56.32^{\circ} \\ 2 \theta_{\text {max }}: & 897,529 \\ N(h k l)_{\text {measured }}, N(h k l)_{\text {unique }}: & I_{\text {obs }}>2 \sigma\left(I_{\mathrm{obs}}\right), 330 \\ \text { Criterion for } I_{\mathrm{obs}}, N(h \mathrm{hl})_{\mathrm{gt}}: & 26 \\ N(\text { param })_{\text {refined }}: & \text { SHELXL-97 [4], DIAMOND [5] } \\ \text { Programs: } & \end{array}$

Acknowledgment. This work was supported by the Deutsche Forschungsgemeinschaft.

\section{References}

1. Gnutzmann, G.; Dorn, F.W.; Klemm, W.: Über einige $\mathrm{A}_{3} \mathrm{~B}$ - und $\mathrm{AB}_{2}$-Verbindungen der schweren Alkalimetalle mit Elementen der $\mathrm{V}$. Gruppe. Z. Anorg. Allg. Chem. 309 (1961) 210-225.

2. Brauer G.; Zintl, E.: Konstitution von Phosphiden, Arsendiden, Antimoniden und Wismuthiden des Lithiums, Natriums und Kaliums. Z. Phys. Chem. B37 (1937) 323-35.

3. Hafner, P.; Range, K.-J.: $\mathrm{Na}_{3}$ As revisited: high-pressure synthesis of single crystals and structure refinenment. J. Alloys Comp. 216 (1984) 7-10.

4. Sheldrick, G. M.: SHELXL-97. A Program for Refining Crystal Structures. University of Göttingen, Germany 1997.

5. Brandenburg, K.: DIAMOND. Visual Crystal Structure Information System Crystal Impact GbR, Bonn, Germany 1997-2002.

Table 2. Atomic coordinates and displacement parameters $\left(\right.$ in $\AA^{2}$ ).

\begin{tabular}{|c|c|c|c|c|c|c|c|c|c|c|}
\hline Atom & Site & $x$ & $y$ & $z$ & $U_{11}$ & $U_{22}$ & $U_{33}$ & $U_{12}$ & $U_{13}$ & $U_{23}$ \\
\hline $\operatorname{As}(1)$ & $6 c$ & 0 & $0.3284(3)$ & $0.3443(4)$ & $0.033(1)$ & $0.0321(7)$ & $0.0305(7)$ & $0.0165(6)$ & 0 & $-0.0009(6)$ \\
\hline $\mathrm{Cs}(1)$ & $6 c$ & $0.6331(2)$ & 0 & $0.51043(9)$ & $0.0544(6)$ & $0.095(1)$ & $0.0329(8)$ & $0.0473(7)$ & $-0.0017(9)$ & 0 \\
\hline $\mathrm{Cs}(2)$ & $6 c$ & $0.7043(2)$ & 0 & $0.1789(1)$ & $0.0654(8)$ & $0.048(1)$ & $0.0323(7)$ & $0.0240(5)$ & $0.0001(9)$ & 0 \\
\hline $\mathrm{Cs}(3)$ & $2 a$ & 0 & 0 & $0.4216(4)$ & $0.0385(7)$ & $U_{11}$ & $0.069(1)$ & $U_{11} / 2$ & 0 & 0 \\
\hline $\mathrm{Cs}(4)$ & $4 b$ & $2 / 3$ & $1 / 3$ & $0.2958(3)$ & $0.0332(5)$ & $U_{11}$ & $0.175(3)$ & $U_{11} / 2$ & 0 & 0 \\
\hline
\end{tabular}

\footnotetext{
* Correspondence author (e-mail: deiseroth@ chemie.uni-siegen.de)
} 